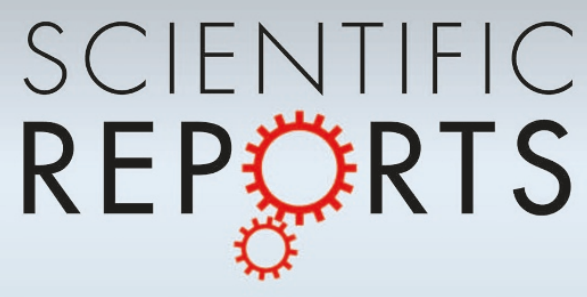

OPEN

SUBJECT AREAS:

QUANTUM MECHANICS

OPTICAL PHYSICS

OPTICS AND PHOTONICS

QUANTUM INFORMATION

Received

22 March 2013

Accepted

5 August 2013

Published

27 August 2013

Correspondence and requests for materials should be addressed to

C.M.L. (cheming.li@ gmail.com) or Y.N.C. (yuehnan@mail.ncku. edu.tw)

\section{Examining non-locality and quantum coherent dynamics induced by a common reservoir}

\author{
Guang-Yin Chen', Shin-Liang Chen ${ }^{2}$, Che-Ming Liं ${ }^{3} \&$ Yueh-Nan Chen ${ }^{2}$
}

\begin{abstract}
'Department of Physics, National Chung Hsing University, Taichung 402, Taiwan, ${ }^{2}$ Department of Physics and National Center for Theoretical Sciences, National Cheng-Kung University, Tainan 701, Taiwan, ${ }^{3}$ Department of Engineering Science and Supercomputing Research Center, National Cheng-Kung University, Tainan City 701, Taiwan.
\end{abstract}

If two identical emitters are coupled to a common reservoir, entanglement can be generated during the decay process. When using Bell's inequality to examine the non-locality, however, it is possible that the bound cannot be violated in some cases. Here, we propose to use the steering inequality to examine the non-locality induced by a common reservoir. Compared with the Bell inequality, we find that the steering inequality has a better tolerance for examining non-locality. In view of the dynamic nature of the entangling process, we also propose to observe the quantum coherent dynamics by using the Leggett-Garg inequalities. We also suggest a feasible scheme, which consists of two quantum dots coupled to nanowire surface plasmons, for possible experimental realization.

pontaneous emission (SE) occurs when a two-level emitter exponentially relaxes into its ground state and is one of the fundamental concepts in quantum mechanics. Its history can be traced back to such early works as that of Albert Einstein. When considering an ensemble of two-level emitters, the emission rate can be greatly enhanced, a phenomenon known as superradiance $(S R)^{1}$. The phenomenon of SR has been widely studied in atomic ${ }^{2}$ and solid state ${ }^{3-5}$ systems. Its role and applications in quantum information science have also been investigated recently ${ }^{6,7}$. The key ingredient in SR is that the emitters exchange excitations with one another via the common reservoir. On one hand, this process creates non-local quantum coherence (entanglement) between the emitters $^{8}$. On the other hand, one should also note that it is a time-dependent process which involves quantum coherent dynamics.

To test the non-locality, the Bell inequality is probably the mostly utilized tool'. More recently, an inequality based on the concept of quantum steering was proposed ${ }^{10}$. This enables one to make a comparison between the hierarchy of states which violate Bell's inequality, quantum steering, and entanglement. Focusing on a single object, Leggett and Garg in 1985 derived an inequality ${ }^{12}$ to test the assumptions of macroscopic realism and the possibility of non-invasive measurement. An experimental violation of this inequality in a superconducting circuit has recently been seen ${ }^{13}$. The Leggett-Garg (LG) inequality can also be applied to microscopic systems ${ }^{14-18}$ to examine the quantum coherent dynamics therein.

The entanglement generated during SR (or reservoir-induced entanglement) is a well-known phenomenon. However, to our knowledge, there are no studies on exactly how well the states generated by SR, in a realistic situation, can violate the hierarchy of inequalities outlined above. Actually, the superradiant process provides a good platform to examine not only non-locality, but also the quantum coherent dynamics. Therefore, we will separately investigate these two quantum features of the simplest superradiant case: SE of two identical emitters coupled to a common environment. As is well known, and will be demonstrated explicitly here, the entanglement, the steering inequality, and the Bell inequality form a logical hierarchy. Since the collective decay is a dynamical process, we then propose to use the LG inequalities ${ }^{12,14,15,19}$ to examine the quantum coherent dynamics. We will show that the LG inequality can be violated when choosing a suitable observable. We will also utilize the recentlydeveloped extension of Leggett-Garg inequality (ELG ${ }^{14,15,19}$ to further study the quantum coherent dynamics. For the experimental realization, we will provide a feasible scheme, which consists of two quantum dots coupled to nanowire surface plasmons ${ }^{20}$. 


\section{Results}

Consider two identical two-level emitters coupled to a reservoir. The interaction Hamiltonian is written as

$$
H_{\text {int }}=\sum_{\vec{k}} \hbar\left[g_{\vec{k}}\left(\sigma_{1}^{+} a_{\vec{k}}+\sigma_{2}^{+} a_{\vec{k}} e^{i \vec{k} \cdot \vec{d}}\right)+\text { H.c. }\right],
$$

where $a_{\tilde{k}}$ is the annihilation operator of the $\vec{k}$-mode field in the reservoir, $\sigma_{j}^{+}$is the raising operator of the $j$ th two-level emitter, and $\vec{d}$ is the inter-emitter distance vector. Here, $g_{\vec{k}}$ is the coupling strength between the $j$ th emitter and the $\vec{k}$-mode field.

In the interaction picture, the evolution of the total system (denoted by the density matrix $\chi$ ) is governed by the Schrödinger equation $\dot{\chi}=\frac{1}{i \hbar}\left[H_{\text {int }}, \chi\right]$. After performing the Born-Markov approximation, the equation of motion of the reduced density matrix $\rho$ in the single excitation manifold reads

$$
\begin{aligned}
\dot{\rho} & =\frac{\gamma}{2} \frac{\operatorname{Sin}(q d)}{q d}\left(2 \sigma_{1}^{-} \rho \sigma_{2}^{+}-\sigma_{2}^{+} \sigma_{1}^{-} \rho-\rho \sigma_{2}^{+} \sigma_{1}^{-}\right) \\
& +\frac{\gamma}{2} \frac{\operatorname{Sin}(q d)}{q d}\left(2 \sigma_{2}^{-} \rho \sigma_{1}^{+}-\sigma_{1}^{+} \sigma_{2}^{-} \rho-\rho \sigma_{1}^{+} \sigma_{2}^{-}\right) \\
& +\sum_{j=1,2} \frac{\gamma}{2}\left(2 \sigma_{j}^{-} \rho \sigma_{j}^{+}-\sigma_{j}^{+} \sigma_{j}^{-} \rho-\rho \sigma_{j}^{+} \sigma_{j}^{-}\right),
\end{aligned}
$$

where $d=|\vec{d}|, \rho(t)=\operatorname{Tr}_{\mathrm{R}}[\chi(t)]$ with R denoting the reservoir, and $\gamma$ is the spontaneous emission rate of the emitter. Here, $q=\omega_{0} / c$ with $\omega_{0}$ being the two-level energy spacing of the emitter and $c$ being the speed of light.

In the single excitation manifold, $\rho(t)$ can be spanned by the basis $\{|1,0\rangle,|0,1\rangle,|0,0\rangle\}$, where $|1,0\rangle(|0,1\rangle)$ denotes the first emitter is in the excited (ground) state with the second one in its ground (excited) state, and $|0,0\rangle$ represents both the emitters are in the ground state. The inset in Fig. 1 shows the quantum coherence $(|1,0\rangle\langle 0,1|)$ between the two emitters in the limit of $d=0$. As seen, the coherence saturates to a fixed value. This quantum coherence, created by the cross terms in the master equation, leads to the entanglement between the two emitters.

Quantum entanglement induced by the common reservoir. To demonstrate the degree of the entanglement, we use $\rho$ to calculate the concurrence ${ }^{21} C=C\left(\rho_{s}\right)=\max \left\{\lambda_{1}-\lambda_{2}-\lambda_{3}-\lambda_{4}\right\}$, where $\lambda_{i}$ s are the square roots of the eigenvalues of $\rho \tilde{\rho}$ with $\tilde{\rho}=\left(\sigma_{y} \otimes \sigma_{y}\right)$ $\rho^{*}\left(\sigma_{y} \otimes \sigma_{y}\right)$. In Fig. 1, we assume two different initial states: $|1,0\rangle$ for Fig. 1(a) and the triplet state $\frac{1}{\sqrt{2}}(|1,0\rangle+|0,1\rangle)$ for Fig. 1(b). As shown by the red-dashed curve in Fig. 1(a), the excitation is transferred to the second emitter with the coherence induced by the common reservoir. The entanglement is created during the excitation transfer and becomes saturated as $t \rightarrow \infty$. However, when the initial state is the triplet one, the entanglement (reddashed curve) decays exponentially with an enhanced rate as shown in Fig. 1(b).

It is interesting to compare the concurrence to the quantum dis$\operatorname{cord}^{22}$, which is a measure of quantum correlations between two subsystems of a quantum system. In our system, if we denote the first (second) emitter as subsystem A (B), the quantum mutual information can be defined as

$$
\mathcal{I}(A: B)=S\left(\rho_{A}\right)+S\left(\rho_{B}\right)-S(\rho)
$$

and its equivalent expression $\mathcal{J}(A: B)$ can be written as

$$
\mathcal{J}_{\left\{\Pi_{i}\right\}}(A: B)=S\left(\rho_{A}\right)-S_{\left\{\Pi_{i}\right\}}(A \mid B) .
$$

The quantum discord $\mathcal{D}(A: B)$ is then defined as ${ }^{22,23}$

$$
\mathcal{D}(A: B)=\mathcal{I}(A: B)-\mathcal{J}(A: B),
$$


Figure $1 \mid$ The concurrence and quantum discord. The concurrence (reddashed curve) and the quantum discord (black-solid curve) of two-emitter superradiance for the initial states: (a) $|1,0\rangle$ and (b) $\frac{1}{\sqrt{2}}(|1,0\rangle+|0,1\rangle)$. The complete set of the projectors $\left\{\Pi_{i}\right\}\left\{\cos \theta|1\rangle+e^{i \phi} \sin \theta|0\rangle, e^{-i \phi} \sin \theta|1\rangle-\right.$ $\cos \theta|0\rangle\}$ is found to contribute maximally to the value of $\mathcal{J}_{\left\{\Pi_{i}\right\}}(A: B)$ for both initial states when $\theta=\frac{\pi}{4}$ (for any $\phi$ ). Inset: The coherence (offdiagonal) term, $|1,0\rangle\langle 0,1|$. In plotting this figure, we have assumed the inter-emitter distance: $d=0$.

where $S(\rho)=-\operatorname{Tr}(\rho \log \rho$ ) (in what follows, we use the natural logarithm) is the von Neumann entropy of the system, $\rho_{A}\left(\rho_{B}\right)$ is the reduced density matrix of $\rho$ after tracing out $B(A)$, and $S_{\left\{\Pi_{i}\right\}}(A \mid B)$ in Eq. (4) is the quantum conditional entropy describing the average uncertainty about the state of $A$ given that the state of $B$ is known. $\mathcal{J}_{\left\{\Pi_{i}\right\}}(A: B)$ is generalized from its classical counterpart through measuring the subsystem $\mathrm{B}$ by a complete set of projectors $\left\{\Pi_{i}\right\}$. Since $\mathcal{J}_{\left\{\Pi_{i}\right\}}(A: B)$ strongly depends on the projectors $\left\{\Pi_{i}\right\}$, the quantity $\mathcal{J}(A: B)$ in Eq. (5) denotes the maximum of $\mathcal{J}_{\left\{\Pi_{i}\right\}}(A: B)$ over all $\left\{\Pi_{i}\right\}$.

We plot the quantum discord by projecting the subsystem $B$ with the complete set $\left\{\cos \theta|1\rangle+e^{i \phi} \sin \theta|0\rangle, e^{-i \phi} \sin \theta|1\rangle-\cos \theta|0\rangle\right\}$ for the initial states $|1,0\rangle$ [Fig. 1(a)] and $\frac{1}{\sqrt{2}}(|1,0\rangle+|0,1\rangle)$ [Fig. 1(b)], 
respectively. As shown by the black-solid curves in Fig. 1, though the quantum discord has been proven to be more robust ${ }^{24}$ than the concurrence in some systems, the behavior of the quantum discord resembles that of concurrence. This is because the entanglement is always present in our system, and thus there is no such thing as, "quantum discord without entanglement" here. In this case, the concurrence and the quantum discord reveal similar behavior.

Testing the non-locality. Let us first utilize the Bell inequality to test the non-local properties of the emitters during the collective decay. The Bell quantity associated with the $\mathrm{CHSH}$ inequality has the following form ${ }^{25}$

$$
\hat{B}_{C H S H} \equiv \hat{a} \cdot \hat{\sigma} \otimes\left(\hat{b}+\hat{b}^{\prime}\right) \cdot \hat{\sigma}+\hat{a}^{\prime} \cdot \hat{\sigma} \otimes\left(\hat{b}-\hat{b}^{\prime}\right) \cdot \hat{\sigma},
$$

where $\hat{a}, \hat{a}^{\prime}, \hat{b}, \hat{b}^{\prime}$ are unit vectors in $\mathbf{R}^{3}$. Here, $\hat{a} \cdot \hat{\sigma} \equiv \sum_{i=1}^{3} a_{i} \sigma_{i}$, where $\sigma_{i}$ is the standard Pauli matrix. Then, the CHSH inequality of a state $\rho$ is

$$
\left|\left\langle\hat{B}_{C H S H}\right\rangle_{\rho}\right|=\left|\operatorname{Tr}\left(\rho \hat{B}_{C H S H}\right)\right| \leq 2 .
$$

The maximum value of the $\mathrm{CHSH}$ inequality is given by

$$
B_{\text {max }}=\max _{\hat{a}, \hat{a}^{\prime}, \hat{b}, \hat{b}^{\prime}} \operatorname{Tr}\left(\rho \hat{B}_{C H S H}\right) .
$$

Assuming a general initial state: $\cos \alpha|1,0\rangle-\sin \alpha|0,1\rangle$, we plot in Fig. 2(a) the maximum value of the $\mathrm{CHSH}$ inequality $\left(B_{\max }\right)$ as
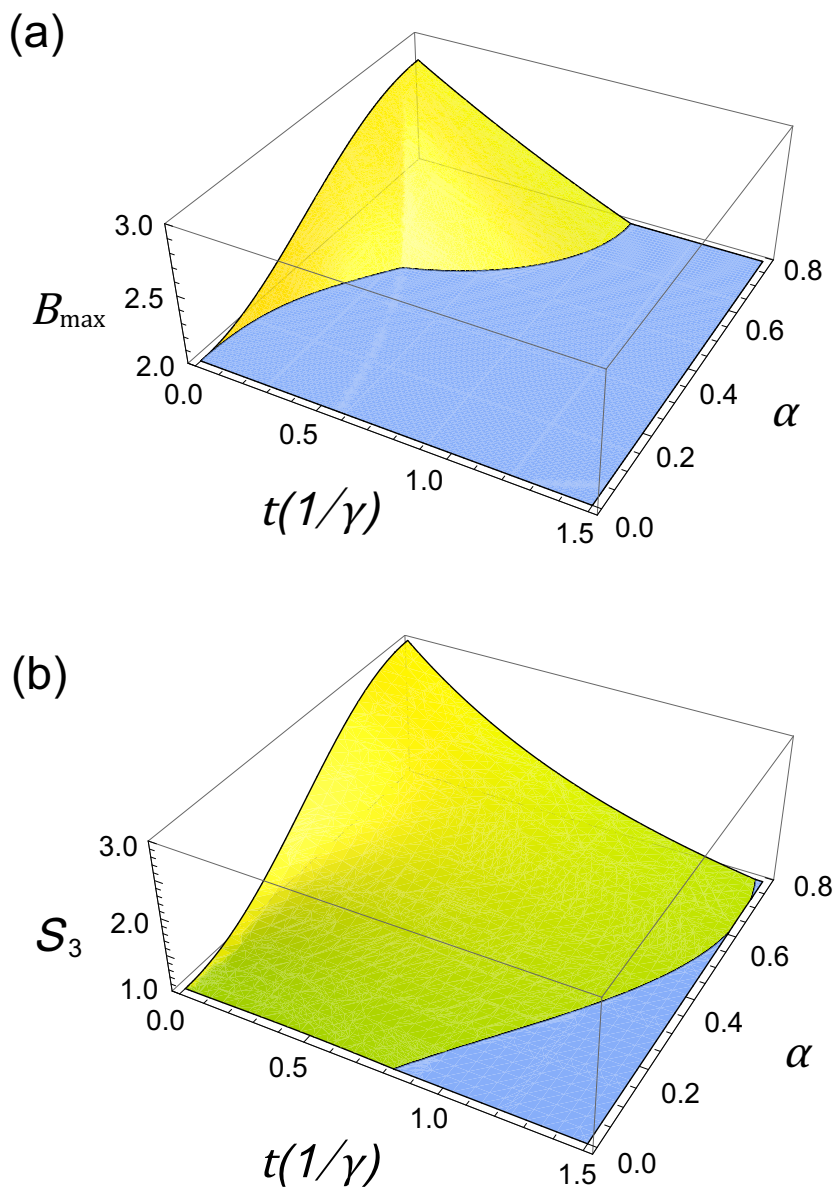

Figure $2 \mid$ Testing the Bell-CHSH and steering inequalities during the superradiant process. Assuming a general initial state: $\cos \alpha|1,0\rangle-$ $\sin \alpha|0,1\rangle$, we plot in (a) the maximum value of the Bell-CHSH inequality and in (b) the steering parameter as functions of time and $\alpha$ in the limit of $d=0$. In plotting the figure, the time $t$ is in unit of $1 / \gamma$. functions of time and $\alpha$ in the limit of $d=0$ for the superradiant process.

As a comparison, let us also consider the steering inequality. If the correlation measurement on the emitter-1 $(B)$ and emitter- $2(A)$ is described by the probability distribution $P\left(B_{i}=b, A_{i}=a\right)$ with $b=$ \pm 1 , and $a= \pm 1$, the steering inequality is written as $^{26}$

$$
S_{N} \equiv \sum_{i=1}^{N} E\left[\left\langle\hat{A}_{i}\right\rangle_{B_{i}}^{2}\right] \leq 1,
$$

where $N(=2$ or 3$)$ is the number of mutually unbiased measurements (the Pauli $\hat{X}, \hat{Y}$ and $\hat{Z}$ ) for the emitter-2, and

$$
E\left[\left\langle\hat{A}_{i}\right\rangle_{B_{i}}^{2}\right] \equiv \sum_{b= \pm 1} P\left(B_{i}=b\right)\left\langle\hat{A}_{i}\right\rangle_{B_{i}=b}^{2}
$$

with emitter-2's expectation value for a measured (conditioned on emitter-1's result) defined as

$$
\left\langle\hat{A}_{i}\right\rangle_{B_{i}=b} \equiv P\left(A_{i}=+1 \mid B_{i}=b\right)-P\left(A_{i}=-1 \mid B_{i}=b\right) .
$$

In Fig. 2(b), we also plot the steering parameter $\left(S_{3}\right)$ as functions of time and $\alpha$ in the limit of $d=0$.

As shown in Fig. 2, we can see that the area of violating the steering inequality is larger than that of violating the Bell inequality. In the limit of $\alpha \rightarrow 0$ (only one of the emitters is excited), Bell's inequality can never be violated, while the steering one can still be violated. This again shows that the steering inequality has better tolerance in examining the non-locality of the entangled states ${ }^{10}$, as expected. Together with the results in Fig. 1, one can see that the entanglement, the steering inequality, and Bell's inequality form a logical hierarchy ${ }^{11}$, i.e. every violation in Bell's inequality leads to the violation of the steering inequality (but not vice versa) and every violation in the steering inequality is a entanglement witness (but not vice versa). The underlying physics of this logic hierarchy can be understood as follows. From the operational definition ${ }^{11}$ of Bell non-locality and steerability, Bell non-locality can be viewed as a three-party task: Alice and Bob, who cannot communicate with each other, try to convince Charlie that the state they share is entangled. Charlie does not trust them and can be convinced only if the correlations between them do not fit the local hidden variable model. However, the steerability is regarded as a two-party task: Alice tries to convince Bob that the state she prepares is entangled, but Bob does not trust her. Bob can be convinced only if the correlations between his local measurements and Alice's results cannot be explained by a local hidden state model. In Bell's non-local scenario, if we relax the condition to make Bob trustworthy, the result will be consistent with that of the steering one. Bell's non-locality is therefore a stronger non-local concept compared with the steerability. In other words, an entangled state, which violates the steering inequality might not violate Bell's inequality. The steering inequality therefore has better tolerance in examining the non-locality.

Testing the quantum coherent dynamics. Given an observable $Q(t)$, which is bound by $|Q(t)| \leq 1$, the assumption ${ }^{12}$ of realism and noninvasive measurability implies the Leggett-Garg inequality ${ }^{12}$ :

$$
L G_{Q} \equiv\left\langle Q\left(t_{1}\right) Q\right\rangle+\left\langle Q\left(t_{1}+t_{2}\right) Q\left(t_{1}\right)\right\rangle-\left\langle Q\left(t_{1}+t_{2}\right) Q\right\rangle \leq 1,
$$

here $Q \equiv Q(t=0)$, and $t_{1}<t_{2}$. The experimental violations of this inequality in a "macroscopic" superconducting circuit ${ }^{13}$, photon polarization states ${ }^{27-29}$, electron-nuclear spin pairs ${ }^{30,31}$ have recently been seen.

Normally, the LG inequality is applied to a macroscopic object to verify the property of macroscopic realism. Here, we wish to make use of it as a tool to check the quantumness (quantum coherent dynamics) during the superradiant process. By choosing $t_{1}=t_{2}=$ $t$ with the initial state being in $|1,0\rangle$, we apply the original LG inequality in Eq. (12) to examine the quantumness in the two-emitter 


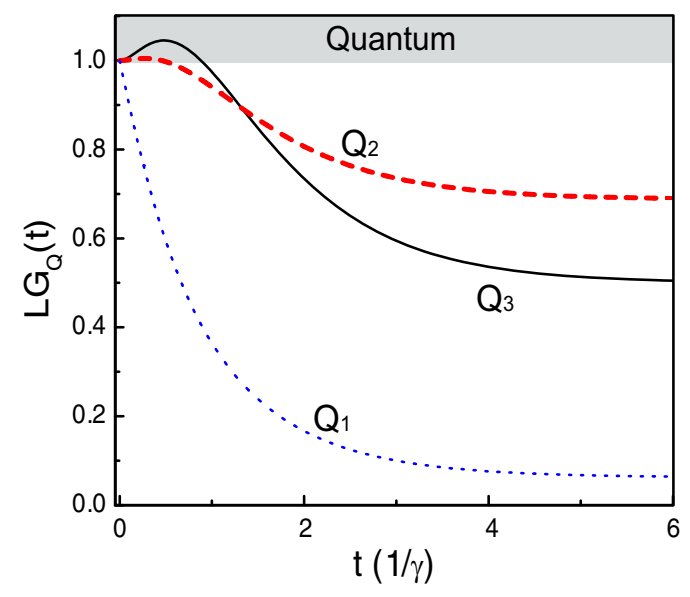

Figure 3 The original LG inequality for as a function of time. The bluedotted, dashed, and solid curves represent the results of the observables $Q=Q_{1}=|1,0\rangle\left\langle 1,0\left|, Q=Q_{2}=\right| 1,0\right\rangle\langle 1,0|+| 0,0\rangle\langle 0,0|$, and $Q=Q_{3}=$ $1-2|0,1\rangle\langle 0,1|$, respectively. In plotting this figure, the initial state is assumed to be $|1,0\rangle$.

SR scenario. If we choose $Q=Q_{1}=|1,0\rangle\langle 1,0|$ as our observable, there is no violation of $L G_{Q}(t)$ as shown by the blue-dotted curve in Fig. 3. This coincides with our intuition: the decay of the state $|1,0\rangle$ is a monotonically decreasing function. However, if we choose $Q=Q_{2}$ $=|1,0\rangle\langle 1,0|+| 0,0\rangle\langle 0,0|$, i.e. the excitation is not in the second emitter, the red-dashed curve shows the violation of $L G_{Q_{2}}(t)$ in the early stage of the time domain. We also plot in Fig. 3 the result of $Q=$ $Q_{3}=1-2|0,1\rangle\langle 0,1|$. One can see that the maximum of the violation $L G_{Q_{3}}(t)$ is enhanced. The analytical results of $L_{Q}(t)$ can also be worked out. For instance, the expression of $L G_{Q_{3}}(t)$ is

$L G_{Q_{3}}(t)=1+\frac{1}{2} e^{-2 \gamma t} e^{-2 \beta}\left[-2 e^{\gamma t} e^{\beta}\left(-1+e^{\beta}\right)^{2}+\left(-1+e^{2 \beta}\right)^{2}\right]$,

where $\beta \equiv \frac{\operatorname{Sin}(q d)}{q d} \gamma t$.

In practice, the non-invasive measurements are not easily implemented. Recently, an extension of the LG inequality was developed for an open transport system ${ }^{14,15}$. Based on the ChapmanKolmogorov equation in stochastic theory ${ }^{14}$, when measuring the population of a state of a classical Markovian system, the following inequality can be derived:

$$
\left|L_{Q}(t)\right| \equiv|2\langle Q(t) Q\rangle-\langle Q(2 t) Q\rangle| \leq\langle Q\rangle,
$$

where $\langle Q\rangle$ is the expectation value of the zero-time population $Q \equiv$ $Q(t=0)$, and $\langle Q(t) Q\rangle$ is the two-time correlation function. Note that if the zero-time state is the steady state then this is equivalent to the original ${ }^{12}$ LG inequality when the measurements are performed noninvasively. Violations of Eq. (14) mean that the dynamics is beyond the classical Markovian description. Note that in deriving Eq. (14) the assumption of the non-invasive measurement is not required in that one can replace the initial measurement by state preparation.

The blue-dotted, red-dashed, and solid curves in Fig. 4(a) represent the results of $\left|L_{Q}(t)\right|$ for the observables $Q=Q_{1}=|1,0\rangle\langle 1,0|, Q$ $=Q_{2}=|1,0\rangle\langle 1,0|+| 0,0\rangle\langle 0,0|$, and $Q=Q_{3}=1-2|0,1\rangle\langle 0,1|$, respectively. Again, we see that the violation depends crucially on the choice of the observable. Figure $4(\mathrm{~b})$ is the $3 \mathrm{D}$ plot of $\left|L_{Q_{3}}(t)\right|$ as functions of time $t$ and inter-emitter distance $q d$. As expected, the maximum of the violation gradually decays to unity when increasing $d$. As $q d=n \pi$, where $n$ is an integer, the function $\left|L_{Q_{3}}(t)\right|$ is not violated because the two emitters act as independent objects due to the interference effect in $\mathrm{SR}^{32,33}$. This can be seen more clear from the analytic expression of $L_{Q_{3}}(t)$.


(c)

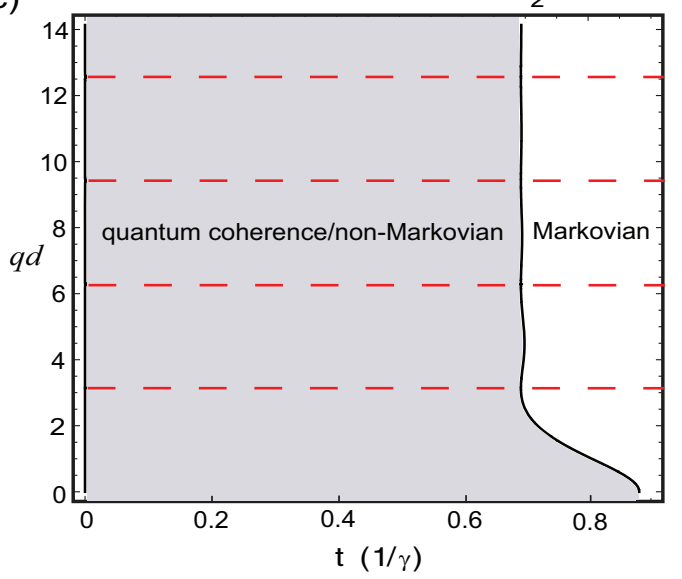

Figure 4 Violations of ELG inequality during the spontaneous decay of two emitters. With the initial state is initially in $|1,0\rangle$, we plot (a) The ELG inequalities for $\left|L_{Q}(t)\right| /\langle Q\rangle$ as a function of time. The blue-dotted, reddashed, and solid curves represent the results of the observables $Q=Q_{1}=$ $|1,0\rangle\left\langle 1,0\left|, Q=Q_{2}=\right| 1,0\right\rangle\langle 1,0|+| 0,0\rangle\langle 0,0|$, and $Q=Q_{3}=1-2|0,1\rangle$ $\langle 0,1|$, respectively. (b) The ELG inequalities for $\left|L_{Q_{3}}(t)\right| /\left\langle Q_{3}\right\rangle$ as functions of time and inter-emitter distance. (c) The border line $\left(\left|L_{Q_{3}}\right|=1\right)$ that separates the violation and non-violation regime. As $q d=n \pi,\left|L_{Q_{3}}\right|$ is equal to unity as shown by the red-dashed lines.

As pointed out in Ref. 14,15, the ELG inequality is equal to the original LG inequality if (i) the zero-time state is the steady state, (ii) the operator $Q$ in Eq. (9) is normalized as $Q=2 Q /\langle Q\rangle-1$, and (iii) the measurements are performed non-invasively. For the observable $Q_{3}$, surprisingly, we find that the kernel is mathematically identical to that of the LG inequality, i.e.,

$$
L_{Q_{3}}(t) /\left\langle Q_{3}\right\rangle=L G_{Q_{3}}(t) .
$$

However, in plotting Fig. 3, we have assumed the excitation is initially in the state $|1,0\rangle$, not the steady state. To answer this, we check the two-time correlation functions and find that $\langle Q(t) Q\rangle=$ $\langle Q(2 t) Q(t)\rangle$ for the observable $Q=Q_{3}$. This explains why the ELG inequality is mathematically equivalent to the original LG inequality. However, one should note that although the mathematical results are the same, it does not mean that they are physically equivalent since the originally LG inequality requires the assumption of non-invasive 
measurement. We can only say that the violation of the ELG inequality is the indication of the quantum coherence.

It is also interesting to see how the time interval of violation $\left(\tau_{\text {vio }}\right)$ varies with inter-emitter distance $q d$. We therefore plot in Fig. 4(c) the border line $\left(\left|L_{Q_{3}}\right|=1\right)$ that separates the violation and non-violation regime. The interval of violation is maximum when $d=0$ and gradually decreases to a fixed value with the superradiant interference feature. To explain the approach to the fixed value, we take the series expansion of Eq. (10) to the order of $\beta^{2} . L_{Q_{3}}$ is then approximated as

$$
L_{Q_{3}}(t) \approx 1+\frac{1}{2} e^{-2 \gamma t}\left(4-2 e^{\gamma t}\right) \beta^{2} .
$$

Therefore, the time interval of violation is $\tau_{\text {vio }}=\ln 2 / \gamma$ in the limit of large $d$. Of course, as $d \rightarrow \infty$, the value of $\left|L_{Q_{3}}\right|$ should go to unity since $\beta \rightarrow 0$ in this limit, i.e. no superradiant effect. Equation (11) is to tell us that $\tau_{\text {vio }}$ is kept fixed, not like the maximum of the violation, when the inter-emitter distance $d$ gradually approaches to infinity.

A proper choice of the observable is the key to see the violation of the ELG inequality. This can be understood by the following example. Consider a qubit in a lossy cavity. Let us choose $\left|+, 0_{c}\right\rangle$ $\left\langle+, 0_{c}\right|$ or $\left|-, 1_{c}\right\rangle\left\langle-, 1_{c}\right|$ as our observables, for which we plot in Fig. 5 the function $\left|L_{Q}\right|$. Here, the state $\left|+, 0_{c}\right\rangle\left(\left|-, 1_{c}\right\rangle\right)$ denotes the qubit is in its excited (ground) state and there is zero (one) photon in the cavity. For the observable $\left|-, 1_{c}\right\rangle\left\langle-, 1_{c}\right|$, it is possible that $\left|L_{Q}\right|$ is not violated (the red-dashed curve) even though the population dynamics is still oscillatory. Therefore, it is very important to choose a proper observable when using the ELG inequality as a tool to indicate the quantumness.

\section{Discussion}

Here, we propose an experimental realization for verifying the quantum coherent dynamics in SR. Consider two quantum dots positioned near a metal nanowire ${ }^{20,34,35}$ as shown in Fig. 6. Due to the quantum confinement, the surface plasmons propagate onedimensionally along the axis direction on the surface of the nanowire. Quantum dot-1 is assumed to be excited initially. In one-dimensional problems, the SR becomes independent of the inter-dot

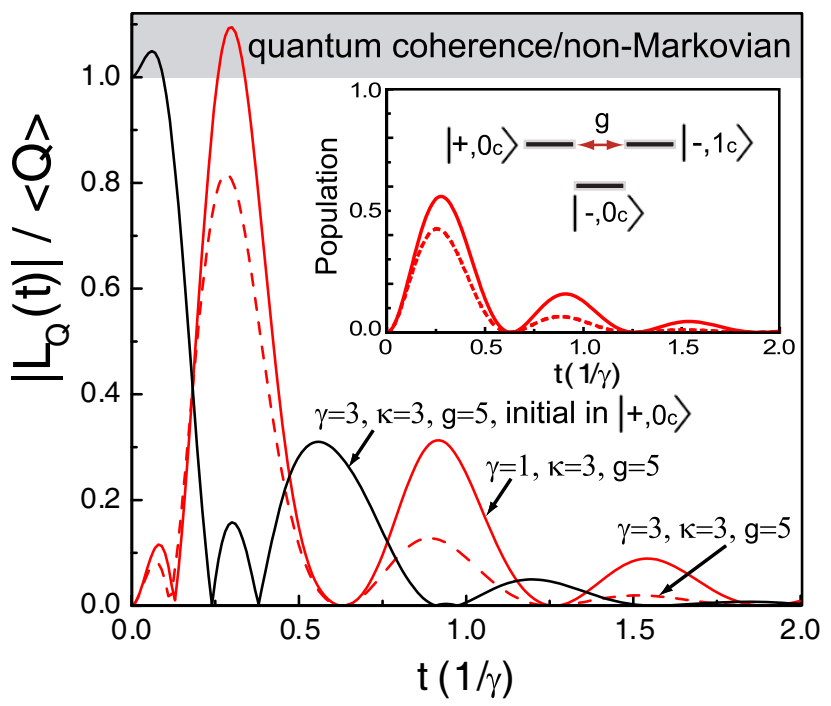

Figure $5 \mid$ The ELG inequality for Rabi-oscillations between a qubit and cavity photon. With the state $\left|+, 0_{c}\right\rangle$ being initially excited, the red curves are the results of the observable $\left|-, 1_{c}\right\rangle\left\langle-, 1_{c}\right|$, while the black curve is the result of the observable $\left|+, 0_{c}\right\rangle\left\langle+, 0_{c}\right|$. Inset: Population dynamics of the state $\left|-, 1_{c}\right\rangle$. Here, $\kappa$ and $\gamma$ are the cavity loss and atomic dissipation rate, respectively, while $g$ is the coupling strength between the qubit and the cavity field.

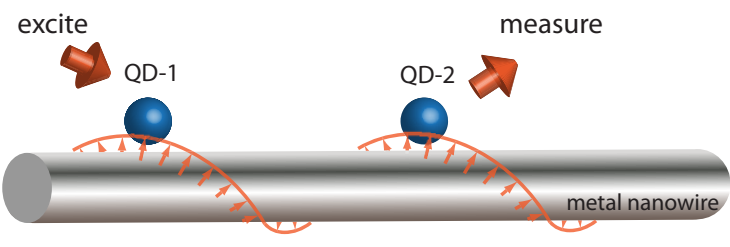

Figure $6 \mid$ Schematics of a experimentally-accessible system. Two twolevel quantum dots coupled to metal nanowire surface plasmons. Here, the quantum dot- 1 is assumed to be excited initially, and one measures the populations of the quantum dot-2.

distance. If we choose $Q=Q_{3}$, the kernel of both LG and ELG inequality can be written as

$$
L_{Q_{3}}(t) /\left\langle Q_{3}\right\rangle=L G_{Q_{3}}(t)=\frac{1}{2}\left(1+e^{-4 \gamma t}-4 e^{-2 \gamma t}+4 e^{-\gamma t}\right) .
$$

The advantage of choosing $Q=Q_{3}=1-2|0,1\rangle\langle 0,1|$ is that, experimentally, one only needs to measure the population of quantum dot-2.

Summarizing, we have utilized both the Bell inequality and the steering inequality to examine the non-locality during the superradiant decay and have shown that the steering inequality has better tolerance in examining the non-locality. Moreover, we have also pointed out that the quantum coherent dynamics during the superradiant decay can be examined by using the LG inequality. Although violating one of the inequalities indicates that quantum effects exist during the superradiant process, the violations have two different meanings: one is for the non-locality (Bell, steering), and the other is for the quantum coherent dynamics (LG). In experiments, we stress that only the measurements on one of the emitters are required to test the LG inequalities. The ELG and LG inequalities are mathematically equivalent when choosing a proper observable. Therefore, the ELG inequality, which does not require the non-invasive measurability, can in practice assist in verifying the quantumness in SR. As a final remark, we wish to point out that the contextuality test ${ }^{36}$ can also be used to indicate the non-locality mentioned above. Similar to the LG inequality, its temporal scenario ${ }^{37}$ can be utilized to indicate the quantum coherent dynamics. Further investigations in this direction are under way.

\section{Methods}

Details of the derivation of Bell-CHSH inequality. To obtain the results in Fig. 2(a), we adopt the method provided in Ref. 26. In order to verify whether the Bell-CHSH inequality is violated for a given state $\rho$, one has to maximize the mean value of the Bell-CHSH parameter:

$$
B_{\text {max }}=\max _{\hat{a}, \hat{a}^{\prime}, \hat{b}, \hat{b}^{\prime}} \operatorname{Tr}\left(\rho \hat{B}_{C H S H}\right)=2 \sqrt{M(\rho)}=2 \sqrt{\lambda_{1}+\lambda_{2}},
$$

where $\lambda_{1}$ and $\lambda_{2}$ are the two largest eigenvalues of $T_{\rho}^{T} T_{\rho}$. Here, $T_{\rho}^{T}$ is the transpose of $T_{\rho}$, and the matrix elements of $T_{\rho}$ is written as

$$
t_{m n}=\operatorname{Tr}\left(\rho \sigma_{m} \otimes \sigma_{n}\right),
$$

where $\sigma_{n}$ is the standard Pauli matrix.

Details of the derivation of steering inequality. To obtain the results in Fig. 2(b), we first measure the state $\rho$ in the $z$-basis, and the probability that Bob obtains \pm 1 is

$$
P\left(B_{i}= \pm 1\right)=\operatorname{Tr}\left(\hat{\Pi}_{z}^{B_{i}= \pm 1} \rho\right) \text {, }
$$

where

$$
\hat{\Pi}_{z}^{B_{i}=+1}=\left(\begin{array}{ll}
1 & 0 \\
0 & 1
\end{array}\right) \otimes\left(\begin{array}{ll}
1 & 0 \\
0 & 0
\end{array}\right),
$$

and

$$
\hat{\Pi}_{z}^{B_{i}=-1}=\left(\begin{array}{ll}
1 & 0 \\
0 & 1
\end{array}\right) \otimes\left(\begin{array}{ll}
0 & 0 \\
0 & 1
\end{array}\right) .
$$

After Bob's measurement, the state is mapped to 


$$
\rho \rightarrow \rho^{\prime}=\frac{\hat{\Pi}_{z}^{B_{i}= \pm 1} \rho \hat{\Pi}_{z}^{B_{i}= \pm 1}}{\operatorname{Tr}\left(\hat{\Pi}_{z}^{B_{i}= \pm 1} \rho\right)} .
$$

With the state $\rho^{\prime}$, the probability that Alice obtains \pm 1 is

$$
P\left(A_{i}= \pm 1 \mid b\right)=\operatorname{Tr}\left(\hat{\Pi}_{z}^{A_{i}= \pm 1} \rho^{\prime}\right) .
$$

With $P\left(B_{i}= \pm 1\right)$ and $P\left(A_{i}= \pm 1 \mid b\right)$, Eqs. (10) and (11) are obtained accordingly. To calculate $S_{3}$ in Eq. (9), one repeats the above procedure by replacing $\hat{\Pi}_{z}^{B_{i}= \pm 1}, \hat{\Pi}_{z}^{A_{i}= \pm 1}$ with $\hat{\Pi}_{x}^{B_{i}= \pm 1}, \hat{\Pi}_{y}^{B_{i}= \pm 1}$ and $\hat{\Pi}_{x}^{A_{i}= \pm 1}, \hat{\Pi}_{y}^{A_{i}= \pm 1}$. One can then have $S_{3}$ in three mutually unbiased basis.

1. Dicke, R. H. Coherence in spontaneous radiation processes. Phys. Rev. 93, 99-110 (1954).

2. Gross, M. \& Haroche, S. Superradiance: An essay on the theory of collective spontaneous emission. Phys. Rep. 93, 301-396 (1982).

3. Chen, Y. N., Chuu, D. S. \& Brandes, T. Current detection of superradiance and induced entanglement of double quantum dot excitons. Phys. Rev. Lett. 90, 166802 (2003).

4. Brandes, T. Coherent and collective quantum optical effects in mesoscopic systems. Phys. Rep. 408, 315-474 (2005).

5. Scheibner, M. et al. Superradiance of quantum dots. Nat. Phys. 3, 106-110 (2007).

6. Chen, Y. N., Li, C. M., Chuu, D. S. \& Brandes, T. Proposal for teleportation of charge qubits via super-radiance. New J. Phys. 7, 172 (2005)

7. Wagner, R. Jr. \& Clemens, J. P. Performance of a quantum teleportation protocol based on temporally resolved photodetection of collective spontaneous emission. Phys. Rev. A 79, 042322 (2009).

8. Braun, D. Creation of entanglement by interaction with a common heat bath. Phys. Rev. Lett. 89, 277901 (2002).

9. Bell, J. S. On the Einstein Podolsky Rosen paradox. Physics 1, 195-200 (1964).

10. Wiseman, H. M., Jones, S. J. \& Doherty, A. C. Steering, entanglement, nonlocality, and the Einstein-Podolsky-Rosen paradox. Phys. Rev. Lett. 98, 140402 (2007).

11. Jones, S. J., Wiseman, H. M. \& Doherty, A. C. Entanglement, Einstein-PodolskyRosen correlations, Bell nonlocality, and steering. Phys. Rev. A 76, 052116 (2007).

12. Leggett, A. J. \& Garg, A. Quantum mechanics versus macroscopic realism: Is the flux there when nobody looks? Phys. Rev. Lett. 54, 857-860 (1985).

13. Palacios-Laloy, A. Experimental violation of a Bell's inequality in time with weak measurement. Nat. Phys. 6, 442-447 (2010).

14. Lambert, N., Emary, C., Chen, Y. N. \& Nori, F. Distinguishing quantum and classical transport through nanostructures. Phys. Rev. Lett. 105, 176801 (2010)

15. Lambert, N., Chen, Y. N. \& Nori, F. Unified single-photon and single-electron counting statistics: From cavity QED to electron transport. Phys. Rev. A 82, 063840 (2010).

16. Li, C. M., Lambert, N., Chen, Y. N., Chen, G. Y. \& Nori, F. Witnessing quantum coherence: from solid-state to biological systems. Sci. Rep. 2, 885 (2012).

17. Chen, G. Y., Lambert, N., Li, C. M., Chen, Y. N. \& Nori, F. Delocalized singlephoton Dicke states and the Leggett-Garg inequality in solid state systems. Sci. Rep. 2, 869 (2012).

18. Emary, C., Lambert, N. \& Nori, F. Leggett-Garg inequalities. arXiv:1304.5133 (2013).

19. Huelga, S. F., Marshall, T. W. \& Santos, E. Proposed test for realist theories using Rydberg atoms coupled to a high-Q resonator. Phys. Rev. A 52, R2497-R2500 (1995).

20. Akimov, A. V. et al. Generation of single optical plasmons in metallic nanowires coupled to quantum dots. Nature 450, 402-406 (2007).

21. Wootters, W. Entanglement of formation of an arbitrary state of two qubits. Phys. Rev. Lett. 80, 2245-2248 (1998).

22. Ollivier, H. \& Zurek, W. Quantum Discord: A measure of the quantumness of the correlations. Phys. Rev. Lett. 88, 017901 (2002).
23. Wang, B., Xu, Z. Y., Chen, Z. Q. \& Feng, M. Non-Markovian effect on the quantum discord. Phys. Rev. B 81, 014101 (2010).

24. Henderson, L. \& Vedral, V. Classical, quantum and total correlations. J. Phys. A 34, 6899-6905 (2001).

25. Horodecki, R., Horodecki, P. \&Horodecki, M. Violating Bell inequality by mixed spin-1/2 states: necessary and sufficient condition. Phys. Lett. A 200, 340-344 (1995).

26. Smith, D. et al. Conclusive quantum steering with superconducting transitionedge sensors. Nat. Commun. 3, 625 (2012).

27. Goggin, M. E. et al. Violation of the Leggett-Garg inequality with weak measurements of photons. Proc. Natl Acad. Sci. 108, 1256-1261 (2011).

28. Dressel, J., Broadbent, C. J., Howell, J. C. \& Jordan, A. N. Experimental violation of two-party Leggett-Garg inequalities with semiweak measurements. Phys. Rev. Lett. 106, 040402 (2011).

29. Xu, J.-S., Li, C.-F., Zou, X.-B. \& Guo, G.-C. Experimental violation of the LeggettGarg inequality under decoherence. Sci. Rep. 1, 101 (2011).

30. Knee, G. C. et al. Violation of a Leggett-Garg inequality with ideal non-invasive measurements. Nat. Commun. 3, 606 (2011).

31. Athalye, V., Roy, S. S. \& Mahesh, T. S. Investigation of the Leggett-Garg inequality for precessing nuclear spins. Phys. Rev. Lett. 107, 130402 (2011).

32. DeVoe, R. G. \& Brewer, R. G. Observation of superradiant and subradiant spontaneous emission of two trapped ions. Phys. Rev. Lett. 76, 2049-2052 (1996).

33. Mokhlespour, S. M., Haverkort, J. E., Slepyan, G., Maksimenko, S. \& Hoffmann, A. Collective spontaneous emission in coupled quantum dots: Physical mechanism of quantum nanoantenna. Phys. Rev. B 86, 245322 (2012).

34. Chen, G. Y., Lambert, N., Chou, C. H., Chen, Y. N. \& Nori, F. Surface plasmons in a metal nanowire coupled to colloidal quantum dots: Scattering properties and quantum entanglement. Phys. Rev. B 84, 045310 (2011).

35. Tame, M. S. et al. Quantum plasmonics. Nat. Phys. 9, 329-340 (2013).

36. Benatti, F., Floreanini, R. \& Romano, R. Complete positivity and entangled degrees of freedom. J. Phys. A 35, 4955 (2002).

37. Markiewicz, M. \& Kaszlikowski, D. Unified approach to contextuality, nonlocality, and temporal correlations. arXiv:1302.3502 (2013).

\section{Acknowledgements}

This work is supported partially by the National Center for Theoretical Sciences and National Science Council, Taiwan, under the grant number NSC 101-2628-M-006-003-MY3, NSC 101-2112-M-006-016-MY3, NSC 103-2911-I-006-301, and NSC 101-2738-M-006-005-.

\section{Author contributions}

G.Y.C. carried out the calculations with help from S.L.C. under the guidance of C.M.L. and Y.N.C. All authors contributed to the interpretation of the work and the writing of the manuscript.

\section{Additional information}

Competing financial interests: The authors declare no competing financial interests.

How to cite this article: Chen, G.Y., Chen, S.L., Li, C.M. \& Chen, Y.N. Examining non-locality and quantum coherent dynamics induced by a common reservoir. Sci. Rep. 3, 2514; DOI:10.1038/srep02514 (2013).

This work is licensed under a Creative Commons AttributionNonCommercial-NoDerivs 3.0 Unported license. To view a copy of this license, visit http://creativecommons.org/licenses/by-nc-nd/3.0 